\title{
Motility study in right sided diverticular disease of the colon
}

\author{
K SUGIHARA, T MUTO, AND Y MORIOKA \\ From the Department of Surgery, University of Tokyo, Tokyo, Japan
}

SUMMARY Intraluminal pressure in the ascending colon of 13 patients with right sided diverticular disease and 10 of normal subjects was studied with catheter-tip transducer inserted through colonoscopes. In the resting state the colonic motility index of patients with diverticular disease was greater than that of the controls. After intravenous injection of neostigmine methylsulphate higher pressure waves were more frequently observed in patients with diverticular disease than the controls, and the colonic motility index of patients was much greater than that of the controls with statistical significance. From these observations it is suggested that high intraluminal pressure and the abnormal motility in the ascending colon plays an important role in the pathogenesis of right sided diverticular disease.

It is well known that in Western countries left sided diverticular disease is common whereas in Asia right sided diverticular disease is more frequent. Pressure studies $^{12}$ in the sigmoid colon together with histologic observations ${ }^{3-6}$ have clarified the pathogenesis of left sided diverticular disease, however, no answer has been given as to the pathogenesis of right sided diverticular disease.

Although there has been no easy means to investigate the right colon physiologically, recent advances in the technique of colonoscopy has enabled us to examine the right colon and to measure the intraluminal pressure with catheter-tip transducers through the colonoscope to investigate the pathogenesis of right sided diverticular disease.

\section{Methods}

SUBJECTS

The motility of the ascending colon was studied in 10 normal subjects and in 13 patients with right sided diverticular disease (Table 1). The normal subjects had no disorders of the colon and rectum, with normal bowel habits and with no abdominal complaint. The patients had mutiple diverticula only in the caecum and ascending colon, seven of them were asymptomatic, four complained of change of bowel habit and abdominal discomfort, and two had

Address for correspondence: Dr K Sugihara, Department of Surgery. University of Tokyo, 7-3-1 Hongo Bunkyo-ku, Tokyo, Japan.

Received for publication: 11 February 1983 a history of acute diverticulitis.

On the day before examination the subjects were permitted to take fluid only, a low residue diet and two kinds of laxatives (magnesium citrate and dicotyl sodium sulphosuccinate) were administered in the evening. A colonoscope was inserted into the caecum under radiographic screening without administration of sedatives or anticholinergics in consideration of their influence on colonic motility. When the scope had reached the caecum the guide wire was introduced through the biopsy channel and the scope was withdrawn carefully, leaving the guide wire in situ under radiographic screening. Thereafter, a polyethylene tube was inserted along the guide wire into the caecum and after the guide wire was withdrawn the catheter-tip transducer was introduced through the tube into the right colon (Fig. 1).

Half an hour was allowed to elapse before the start of recording in order to minimise the influence of colonoscopy. The motility of the ascending colon

Table 1 Number, sex, and age controls and patients with right sided diverticular disease

\begin{tabular}{lllllll}
\hline & & \multicolumn{2}{l}{ Sex } & & \multicolumn{2}{l}{ Age (year) } \\
\cline { 6 - 7 } \cline { 6 - 7 } & $\begin{array}{l}\text { Subjects } \\
\text { (no.) }\end{array}$ & $M$ & $F$ & & Mean & Range \\
\hline Rt div dis & 13 & 10 & 3 & & $48 \cdot 9$ & $18-78$ \\
Control & 10 & 4 & 6 & & $55 \cdot 2$ & $29-66$ \\
\hline
\end{tabular}


Fig. 1 Method of setting Mikro-Tip transducer in ascending colon using colonoscope.

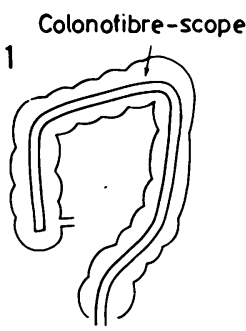

4

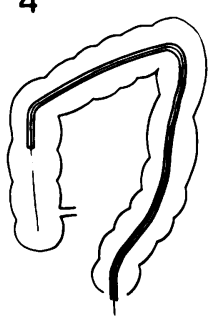

2

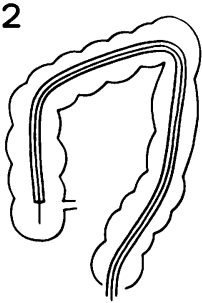

5

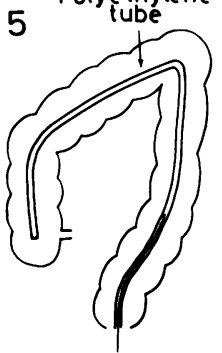

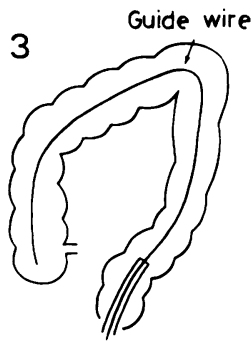

6

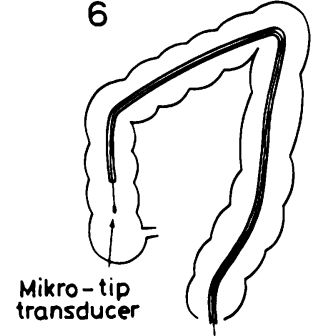

was recorded at rest for 30 minutes (resting state) and then $0.5 \mathrm{mg}$ of neostigmine methylsulphate was given intravenously and the motility was recorded for 30 minutes (neostigmine-stimulated state). During these studies subjects were kept lying on their backs.

The pressure sensor of Mikro-Tip transducer (Millor, Houston, Texas, USA) is located at the tip of the catheter (Fig. 2). This pressure sensor provides high fidelity recording of intraluminal pressure without errors in phase and amplitude because the sensor at the catheter tip changes the pressure directly into the electrical activity and it is easily prepared.

The colonic motility was analysed with seven parameters; (1) the colonic motility index, being the product of the percentage duration of activity and the mean amplitude, (2) the percentage duration of activity (sum of waves' duration $\times 100 /$ total duration of the recording period), (3) the mean
Fig. 2 Mikro-Tip transducer has pressure sensor at tip of catheter and changes the pressure directly into electrical activity. 
amplitude, (4) the number of all waves, (5) the number of waves exceeding $20 \mathrm{mmHg}$, (6) the number of waves exceeding $50 \mathrm{mmHg}$. (7) the mean duration. Waves of $5 \mathrm{mmHg}$ or less were excluded from these calculations because they were sometimes difficult to distinguish from respiratory movements and they would give little influence on seven parameters if they were included in these calculations.

In order to avoid divergency of interpretation between different investigators, all calculations were carried out by one of the authors.

Results were expressed as the mean \pm SE. Statistical analysis was performed by the MannWhitney $\mathrm{U}$ test and the Wilcoxon $\mathrm{T}$ test.

\section{Results}

Figure 3 shows the intraluminal pressure in the ascending colon in a normal subject (upper tracing) and in a patient with right sided diverticular disease (lower tracing). Each tracing represents five minutes of recording time. In the resting state (left half) a few pressure waves can be seen both in the normal subject and in the patient with diverticular disease; however, after neostigmine injection (right half) many high pressure waves are generated successively. In particular, the patient with diverticular disease shows many waves more than $80 \mathrm{mmHg}$ which are absent in the normal subject.

In the resting state the mean values of six parameters except the mean duration of patients with diverticular disease were greater than those of the controls, and the differences in the colonic motility index and in the number of waves exceeding $20 \mathrm{mmHg}$ were statistically significant (Table 2, Fig.
Table 2 Comparison of seven parameters in the resting state between controls and patients with right sided diverticular disease (mean $\pm S E$ )

\begin{tabular}{lccl}
\hline & Control & Rt div dis & $p$ \\
\hline CMI & $37 \cdot 6 \pm 13 \cdot 6$ & $194 \cdot 8 \pm 83 \cdot 5$ & $<0 \cdot 05$ \\
Per dur act & $4 \cdot 3 \pm 1 \cdot 5$ & $9 \cdot 9 \pm 2 \cdot 6$ & - \\
Mean amp & $8 \cdot 3 \pm 1 \cdot 4$ & $14 \cdot 8 \pm 3 \cdot 1$ & - \\
No. all waves & $7 \cdot 3 \pm 2 \cdot 6$ & $16 \cdot 4 \pm 4 \cdot 2$ & - \\
$>20 \mathrm{mmHg}$ & $0 \cdot 2 \pm 0 \cdot 1$ & $4 \cdot 5 \pm 2 \cdot 3$ & $<0 \cdot 05$ \\
$>50 \mathrm{mmHg}$ & 0 & $1 \cdot 5 \pm 1 \cdot 0$ & - \\
Mean duration & $9 \cdot 2 \pm 2 \cdot 4$ & $9 \cdot 0 \pm 1 \cdot 7$ & - \\
\hline
\end{tabular}

4). Neostigmine significantly increased frequency and amplitude of waves both in the controls and in patients with diverticular disease. The mean duration in both groups was slightly longer than after neostigmine stimulation than in the resting state.

After neostigmine stimulation the percentage duration of activity in patients with diverticular disease was $54 \%$ compared with $28.7 \%$ in the controls and the mean amplitude in patients with diverticular disease was $35.4 \mathrm{mmHg}$ compared with $15.5 \mathrm{mmHg}$ in the controls (Table 3 ). The differences between both groups in these parameters were statistically significant. The colonic motility index increased to 470.9 in the controls and to $2,038.6$ in patients with diverticular disease, being more than 10 times as great as in the resting state (Table 3, Fig 4). The colonic motility index in patients with diverticular disease in the neostigmine-stimulated state was significantly greater than that in the controls $(p<0 \cdot 005)$.

In the neostigmine-stimulated state the mean
Fig. 3 Intraluminal pressure patterns in ascending colon. Upper left, resting state of normal subject; upper right. neostigmine-stimulated state of normal subject; lower left, resting state of patient with right sided diverticular disease; lower right, the neostigminestimulated state of patient with right sided diverticular disease. Each tracing represents five minutes of recording time. Many waves exceeding 80 $\mathrm{mmHg}$ are found in tracing of patient after neostigmine stimulation.

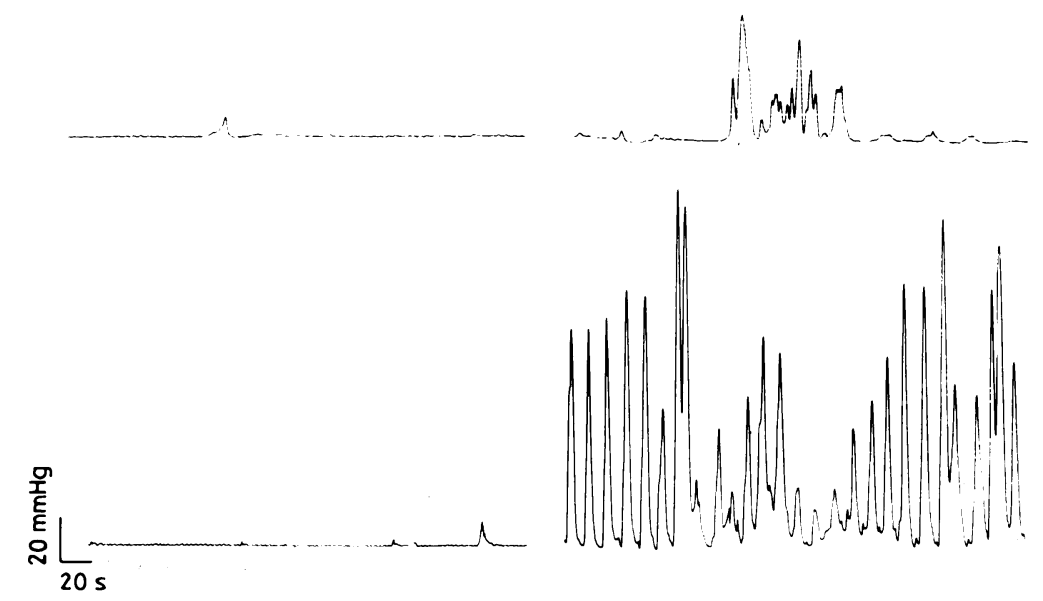




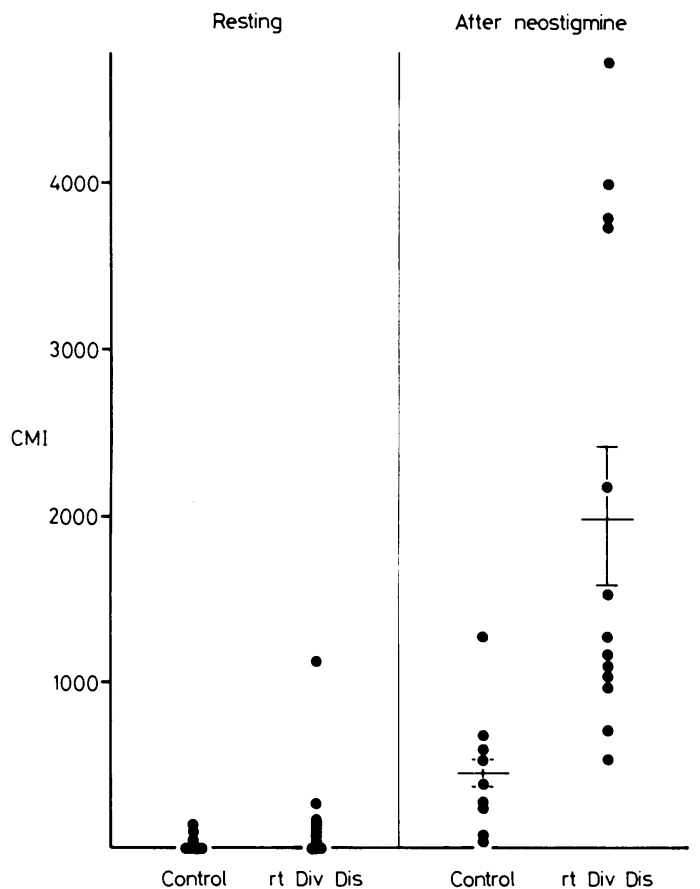

Fig. 4 Colonic motility index in resting state and in neostigmine-stimulate state. Mean colonic motility index of patients with right sided diverticular disease in neostigminestimulate state is much greater than that of others.

Horizontal lines $=$ mean and $S E$.

colonic motility index of seven asymptomatic patients with diverticular disease was $1,792.7$ and that of six symptomatic patients was $2,342 \cdot 2$, suggesting that there was no relationship between clinical symptoms and the colonic motility index. The colonic motilty index of asymptomatic patients was greater than that of the controls with statistical significance $(\mathrm{p}<0.05)$.

The number of all waves were correlated with the percentage duration of activity because there was

Table 3 Comparison of seven parameters in the neostigmine-stimulated state between controls and patients with right sided diverticular disease (mean $\pm S E$ )

\begin{tabular}{lccl}
\hline & Control & Rt div dis & $p$ \\
\hline CMI & $470.9 \pm 1114.0$ & $2,038.6 \pm 417.4$ & $<0.005$ \\
Per dur act & $28 \cdot 7 \pm 5.4$ & $54.0 \pm 6.6$ & $<0.05$ \\
Mean amp & $15.5 \pm 1.6$ & $35.4 \pm 4.0$ & $<0.005$ \\
No. all waves & $37.4 \pm 6.4$ & $96.8 \pm 13.8$ & $<0.005$ \\
$>20$ mmHg & $8 \cdot 3 \pm 2.6$ & $59.9 \pm 12.6$ & $<0.005$ \\
$>50$ mmHg & $0.8 \pm 0.5$ & $25.5 \pm 8.9$ & $<0.005$ \\
Mean duration & $13.1 \pm 1.1$ & $11.3 \pm 1.3$ & - \\
\hline
\end{tabular}

little difference between the duration of each wave. The number of all waves in patients with diverticular disease was 96.8 compared with 37.4 in the controls. Sixty-nine per cent of all waves in patients with diverticular disease were waves exceeding 20 $\mathrm{mmHg}$, whereas it was only $22.2 \%$ in the controls. Although the number of waves exceeding $50 \mathrm{mmHg}$ were few in the controls, they averaged 25.5 during 30 minutes in patients with diverticular disease (Table 3 ). In nine out of 13 patients neostigmine generated waves exceeding $100 \mathrm{mmHg}$. None was found in the controls.

\section{Discussion}

It is well known that in Western countries left sided diverticular disease is common. Painter and Truelove $^{1}$ studied the motility in the sigmoid colon by the open-tip method. Although in the resting state there was no significant difference in the intraluminal pressure between normal subjects and patients with left sided diverticular disease, administration of prostigmine (neostigmine methylsulphate) increased frequency and amplitude of pressure waves and the response was greater in the diseased segments than in the normal sigmoid colons. They suggested that the different response of the affected part of the sigmoid colon was responsible for the pathogenesis and progression of diverticular disease. Arfwidson ${ }^{2}$ also measured the intraluminal pressure in the sigmoid colon under three different conditions; in the resting state, after prostigmine stimulation and postprandially. Total pressure and the number of strong waves in the patients with left sided diverticular disease were significantly greater than those in normal subjects under all three different conditions. He assumed that an increased sigmoid motor activity was of aetiological significance to diverticular disease and the conditions which aggravated the diseased state occurred several times daily in response to food intake.

Slack ${ }^{5}$ reported characteristic sites of herniation were at the points of penetration of intramural vessels and Meyers ${ }^{\mathfrak{t}}$ confirmed it by a combination of pathologic and angiographic examinations of operative and postmortem specimens.

From these observations it is suggested that high intraluminal pressure generated by the abnormal motility of the sigmoid colon facilitates herniation of the colonic mucosa through the circular muscle layer at the points where the vessels penetrate the colonic wall.

In Japan, right sided diverticular disease is more frequent than the left sided one; however, no answer has been given as to the pathogenesis of right 
sided diverticular disease. Recent advances in the technique of colonoscopy made possible a study of the intraluminal pressure in the ascending colon with Mikro-Tip transducer.

The colonic motility index, which was the same as 'total activity' described by Connell. ${ }^{7}$ is perhaps the most significant parameter ${ }^{8}$ because it includes the two important components of motility - that is, the percentage duration of activity and the mean amplitude. In the resting state the colonic motility index of patients with right sided diverticular disease was greater than that of the controls, and after neostigmine stimulation the colonic motility of patients with diverticular disease was much greater than that of the controls. Waves more than 100 $\mathrm{mmHg}$ were not infrequently seen in the diseased segments in the neostigmine-stimulated state. Fujita ${ }^{9}$ and Sasaki ${ }^{10}$ reported that patients with right sided diverticular disease showed high intraluminal pressure in the ascending colon after neostigmine injection, but their studies were not statistically compared between normal subjects and patients with diverticular disease.

There was little difference in the colonic motility between asymptomatic and symptomatic patients with right sided diverticular disease. Asymptomatic patients showed higher intraluminal pressure than the controls. It can be assumed that there is no direct relationship between symptoms and high intraluminal pressure.

It has been believed that diverticula of the caecum and ascending colon were the congenital or true diverticula with all muscular coats in their walls. ${ }^{11}$ Histological studies ${ }^{41213}$ of right sided diverticular disease, however, have pointed out that diverticula of the caecum and ascending colon are false diverticula which consisted of mucosa with surrounding muscularis mucosa, and the circular muscle is conspicuously absent from the diverticular wall. Hughes ${ }^{+}$has said that "the "congenital" caecal diverticulum is largely a pathological myth.'

From our intraluminal pressure study of the ascending colon it is suggested that high intraluminal pressure and the abnormal motility in the ascending colon plays an important role in the pathogenesis of right sided diverticular disease. Further histological study is required in order to clarify the pathogenesis of right sided diverticular disease.

\section{References}

1 Painter NS, Truelove SC. The intraluminal pressure patterns in diverticulosis of the colon. Gut 1964; 5: 365-9.

2 Arfwidson S. Pathogenesis of multiple diverticula of the sigmoid colon in diverticular disease. Acta Chir Scand 1964; suppl 342.

3 Morson BC. The muscle abnormality in diverticular disease of the sigmoid colon. Br J Radiology 1963; 36: 385-92.

4 Hughes LE. Postmortem survey of diverticular disease of the colon. Gut 1969; 10: 336-51.

5 Slack WW. The anatomy, pathology and some clinical features of diverticulosis of the colon. Br J Surg 1962; 50: 185-90.

6 Meyers MA, Volberg F, Katzen B, Alonso D, Abbot G. The angioarchitecture of colonic diverticula. Radiology 1973; 108: 249-61.

7 Connell AM. The motility of the pelvic colon. 1: Motility in controls and in patients with asymptomatic duodenal ulcer. Gut 1961; 2: 175-80.

8 Meunier P, Rochas A, Lambert R. Motor activity of the sigmoid colon in chronic constipation: comparative study with normal subjects. Gut 1979; 20: 1095-101.

9 Fujita K, Watanabe M, Odawara M, et al. A study of colonic diverticulum from the point of its function. Stomach Intestine 1980; 15: 817-23.

10 Sasaki D, Nakazima H, Munakata A, Yoshida U. Intraluminal pressure of diverticular disease and irritable bowel syndrome. Proceedings of 23rd Japanese congress on gastroenterology. Tokyo, Japanese J Gastroenterology 1981: 228.

11 Wagner DE, Zollinger RW. Diverticulitis of the cecum and ascending colon. Arch Surg 1961; 83: 124-31.

12 Williams KL. Acute solitary ulcer and acute diverticulitis of the cecum and ascending colon. Br J Surg 1960; 47: 351-8.

13 Perry PM, Morson BC. Right-sided diverticulosis of the colon. Br J Surg 1971; 58: 902-4. 